\title{
Weaning and extubation
}

\section{Introduction}

The aim of this guideline is to ensure that information regarding the care of patients who require weaning from mechanical ventilation is available to all staff within the Intensive Care Unit (ICU).

\section{Purpose}

These guidelines have been developed in order to assist medical and nursing staff in caring for patients requiring weaning from mechanical ventilation according to evidence-based practice.

\section{Scope}

This guideline applies to all medical, respiratory therapy and nursing staff in the ICU.

\section{Definition}

Weaning covers the entire process of liberating the patient from mechanical support and from the endotracheal tube (ET). Time spent in the weaning process typically represents $40-50 \%$ of the total duration of mechanical ventilation (Boles et al, 2007). Nurses are required to assess the patient's readiness to wean against a number of prerequisites (Crocker, 2009).

\section{Responsibilities}

This Guideline applies to all medical respiratory therapy and nursing staff working in the ICU.

\section{Procedure}

The respiratory therapist, nursing and medical team decide collaboratively if the patient is ready for weaning.

\section{Weaning process: assessment criteria}

The timing of extubation should be carried out when the patient has reached the below assessment criteria. The pace of weaning should be determined by clinical assessment. Keep the respiratory therapist, charge nurse and medical team informed and in agreement on the patient's weaning and extubation plan.

\section{Neurological}

i. Sedation is discontinued

ii. Patient is awake, alert and orientated, able to move all limbs, obeying commands. Patient should have GCS of $>8$.

iii. Pain is well controlled, intravenous analgesia may continue providing all neurological criteria are met

iv. Patient is educated on the extubation process

\section{Respiratory}

i. The patient's breathing is unlabored, equal air entry, not using accessory muscles, respiratory rate normal, spontaneous breathing INDEX $<100$

ii. PS/CPAP ventilation mode, FIO2 requirements 35\%-40\%, PEEP $5, \mathrm{PS}<10$, Spo $2>95 \%$. P $/$ F ratio $>200$
Volume 2 Issue 3 - 2015

Thiruppathi Chockalingam
Hamad Medical Corporation, Qatar

Correspondence: Thiruppathi Chockalingam, Acting Assistant Director of Respiratory Therapy, Hamad Medical Corporation, 3050 Doha, Qatar, Tel (+974) 553I 902 I, (+974) 44392376, Fax (+974) 44391829, Email tchockalingam@hamad.qa

Received: March 12, 20I5 | Published: April 02, 2015

iii. Acid/base balanced, Arterial blood gas (ABG) acceptable for the patient, nebulizers administered if required

iv. Gag/cough reflex present on suctioning/able to protect airway, secretions are acceptable

v. Check in the patient's medical notes that intubation was not difficult

vi. Perform leak test to determine if patient has laryngeal oedema.

vii. All Criteria has been met following spontaneous breathing trial. (SBT)

\section{Cardiovascular}

i. The patient is haemo dynamically stable, maintaining adequate mean arterial pressure (MAP), minimal isotropic support

ii. Hemoglobin $>7$

iii. Minimal chest drain output

iv. No significant ECG changes.

\section{Extubation}

1. Ensure the patient is sitting upright in the bed unless contraindicated by patient's condition.

2. Ensure bedside emergency equipment is available and in working order, including re-intubation equipment.

3. The extubation procedure is a two person procedure Respiratory therapist/nurse

4. Prepare equipment required for extubation:

a. Suction equipment including yankeur suction and suction catheters

b. $60 \%$ FIO2 Face Mask oxygen supply and equipment to deliver humidified oxygen

c. $10 \mathrm{ml}$ syringe

5. Explain the procedure to the patient and reassure if anxious

6. Aspirate naso gastric tube if in situ 
7. Prior to suctioning pre-oxygenate the patient on the ventilator

8. Suction the ET tube as per unit procedure/sub glottic

9. The assisting nurse removes the tape securing the ET tube

10. While the respiratory therapist deflates the ET cuff with $10 \mathrm{ml}$ syringe

11. And removes with a single smooth action. Turn off the ventilator

12. Immediately place patient on $60 \%$ FI02 via face mask

13. Encourage the patient to cough up any remaining oral secretions and suction with yankeur

14. Observe Spo2s, maintain saturations $>95 \%$

15. Perform ABGs within first half hour post extubation

16. Respiratory therapist/nurse to monitor patient closely for 48 hours post extubation. (If required NIV as per hospital protocol.

17. Observe patient closely for potential deterioration or complications

\section{Potential complications during extubation}

i. Laryngeal spasm

ii. Regurgitation/aspiration

iii. Vagal stimulation arrhythmias and cardiac arrest

iv. Trauma

\section{Potential complications following extubation}

i. Hoarseness

ii. Vocal cord paralysis

iii. Dysphagia

iv. Aspiration

v. Laryngeal stenosis, oedema, granuloma vi. Tracheal stenosis at the site of the cuff or the tube lip

vii. Tracheomalacia

viii. Respiratory failure

ix. Lung collapse

x. Cardiopulmonary arrest ${ }^{1-8}$

\section{Acknowledgements}

None.

\section{Conflict of interest}

The author declares no conflict of interest.

\section{References}

1. Burns KE, Meade MO, Lessard MR, et al. Wean Earlier and Automatically with New technology (the Wean study): a protocol of a multicentre, pilot randomized controlled trial. Trails. 2009;10:81.

2. Chen HH, YuJM, Wang LL, et al. Discussion of the risk factors of unplanned removal of endotracheal tube. Journal of Health Science. 2000;2:250-258.

3. Cocker C. nurse led weaning from ventilatory and respiratory support. Intensive Crit Care Nurs. 2002;18(5):272-279.

4. Crocker C, Scholes J. The importance of knowing the patient in weaning from mechanical ventilation. Nurs Crit Care. 2009;14(6):289-296.

5. Henneman EA. Liberating patients from mechanical ventilation. A team approach. Crit Care nurse. 2001;21(3):25-32.

6. Price AM. Nurse-led weaning from mechanical ventilation: where's the evidence? Intensive Crit Care Nurs. 2001;17(3):167-176.

7. Salipante DA. Developing a multidisciplinary weaning unit through collaboration. Crit Care nurse. 2002;22(4):30-39.

8. MacIntyre NR, Cook DJ, Ely EW, et al. Evidence -based guidelines for weaning and discontinuing ventilator support: a collective task force facilitated by the ACCP, AARC, and the ACCCM. Chest. 2001;120(6):375-395. 